\title{
MAP kinase and CAMP signaling regulate infection structure formation and pathogenic growth in the rice blast fungus Magnaporthe grisea
}

\author{
Jin-Rong Xu and John E. Hamer ${ }^{1}$ \\ Department of Biological Sciences, Purdue University, West Lafayette, Indiana 47907 USA
}

\begin{abstract}
Many fungal pathogens invade plants using specialized infection structures called appressoria that differentiate from the tips of fungal hyphae contacting the plant surface. We demonstrate a role for a MAP kinase that is essential for appressorium formation and infectious growth in Magnaporthe grisea, the fungal pathogen responsible for rice blast disease. The PMK1 gene of M. grisea is homologous to the Saccharomyces cerevisiae MAP kinases FUS3/KSS1, and a GST-Pmk1 fusion protein has kinase activity in vitro. pmk1 mutants of $M$. grisea fail to form appressoria and fail to grow invasively in rice plants. pmk1 mutants are still responsive to cAMP for early stages of appressorium formation, which suggests Pmk1 acts downstream of a cAMP signal for infection structure formation. PMK1 is nonessential for vegetative growth and sexual and asexual reproduction in culture. Surprisingly, when expressed behind the GAL1 promoter in yeast, PMK1 can rescue the mating defect in a fus 3 kss 1 double mutant. These results demonstrate that PMK1 is part of a highly conserved MAP kinase signal transduction pathway that acts cooperatively with a cAMP signaling pathway for fungal pathogenesis.
\end{abstract}

[Key Words: Appressorium; PMK1; pathogenicity]

Received July 29, 1996; revised version accepted September 18, 1996.

The most economically devastating disease of cultivated rice, rice blast disease, is caused by the filamentous fungus Magnaporthe grisea (Ou 1985). In spite of the availability of resistant crop genotypes, disease epidemics continue to occur worldwide and threaten food supplies in many emerging nations (Teng 1994). The blast fungus attacks all above-ground parts of the rice plant, and seedlings can be killed during epidemics (Ou 1985). In mature plants, the fungus attacks the emerging seed panicle, resulting in the complete loss of all rice seeds (Ou 1985). As rice remains the major food crop for more than a third of the world's population, improved methods for controlling this disease are needed (Ford et al. 1994). Molecular genetic studies in the blast fungus (Valent 1990) are being used to define key steps in attachment, penetration, and pathogenic growth as targets for developing new antifungal chemicals.

$M$. grisea infects rice plants in a manner typical of other foliar pathogens (see Fig. 1). Asexual spores, called conidia, are dispersed in moist air and attach tightly to the leaf surface (Hamer et al. 1988). In a drop of water, a conidium produces a germ tube that grows and differentiates a specialized infection structure called an appres-

${ }^{1}$ Corresponding author. sorium that adheres tightly to the plant surface (Bourett and Howard 1990). This specialized cell generates enormous turgor pressure that is used to penetrate the underlying plant surface (Howard et al. 1991). Once inside the plant, bulbous, lobed infection hyphae grow in and between plant cells. Eventually a lesion develops that under conditions of high humidity will yield mycelia that sporulate and release more conidia to reinitiate the infection cycle.

Fungal pathogens like $M$. grisea commonly rely on thigmotropic sensing mechanisms to decipher surfaces appropriate for appressorium formation (Hoch and Staples 1991). The rice blast fungus senses the presence of hydrophobic surfaces that mimic the rice leaf surface (Hamer et al. 1988; Bourett and Howard 1990). On hydrophobic surfaces such as Teflon membranes, appressoria are formed shortly after conidial germination, whereas on hydrophilic surfaces, such as glass or agar media, germinating conidia produce long branched hyphae, typical of filamentous fungi (Lee and Dean 1994). Although thigmotropic sensing is poorly understood biochemically, appressorium formation appears to represent a form of cellular morphogenesis requiring the synthesis of specific gene products (Lee and Dean 1993b; Talbot et al. 1993) and cell wall layers (Bourett and Howard 1990). Pharmacological studies in a variety of fungal plant and 


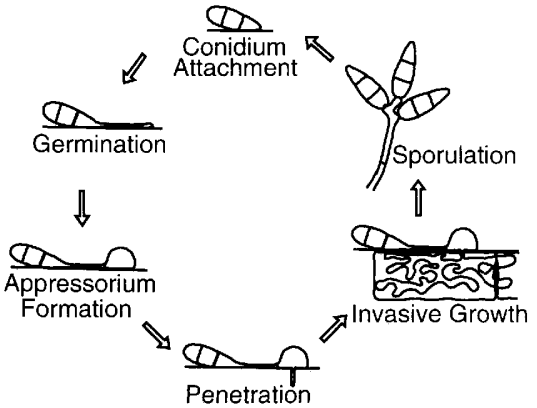

Figure 1. The plant infection cycle of the rice blast fungus. Conidia attach to the rice leaf surface and produce germ tubes that differentiate into dome-shaped appressoria. The appressoria penetrate the plant surface and produce infectious hyphae. The hyphae within the lesions can sporulate under appropriate conditions.

insect pathogens that form appressoria, including $M$. grisea, suggest that conserved signal transduction pathways such as cAMP signaling are required for infection structure formation (St. Leger et al. 1990; Lee and Dean 1993a). cAMP signaling appears to be important for surface recognition in $M$. grisea. Exogenous cAMP stimulates appressorium formation on hydrophilic surfaces, and disruption of a gene related to cAMP-dependent protein kinases $(C P K A)$ causes a delay in appressorium formation (Mitchell and Dean 1995). Pathways acting downstream of the cAMP signal remain to be identified.

Here we show that $M$. grisea contains a mitogen-activated protein (MAP) kinase gene, PMK1 (Pathogenicity MAP-Kinase 11, that most closely resembles the yeast pheromone signaling MAP kinase genes, FUS3 and KSS1. PMK1 can functionally substitute for the Fus3/ Kss1 kinases in yeast. Gene replacement mutants show that PMK1 is essential for appressorium formation and pathogenesis but dispensable for all other aspects of the $M$. grisea life cycle. PMK1 is required for later steps in appressorium formation and may act downstream of the cAMP signaling pathway. Surprisingly, $P M K 1$ is also essential for survival or growth in rice plants. These results show that cooperative signaling between CAMP and a conserved MAP kinase pathway regulates key steps in fungal pathogenesis.

\section{Results}

PMK1 encodes a functional protein kinase related to MAP kinases

A PCR-based screen was used to clone putative MAP kinase homologs from a wild-type strain of $M$. grisea designated Guyl1. DNA sequence analysis identified PCR clones homologous to the yeast FUS3/KSS1 MAP kinases and these clones were used to obtain full-length cDNA and genomic clones (see Materials and Methods). Five cDNA clones with different $5^{\prime}$ and $3^{\prime}$ ends but identical coding regions (A1, A2, A3, A4, and A5; Fig. 2A) were obtained. Sequencing the cognate genomic clone confirmed the origin of all cDNAs and revealed the presence of two small introns with consensus fungal splice sites (Fig. 2A). The open reading frame has the potential to encode a 356 -amino-acid protein with $60.5 \%$ identity and $77.9 \%$ similarity with yeast (Saccharomyces cerevisiae) Fus3, $63.7 \%$ identity and $79.4 \%$ similarity with yeast Kss1, and $55.9 \%$ identity and $72.7 \%$ similarity with human Erk1 MAP kinases (Fig. 2B). Southern blot analysis showed that the cDNA and genomic clones defined a single copy gene (data not shown) that we designated $P M K 1$. The PMK1 coding region contains all 11 conserved protein kinase subdomains and the characteristic MAP kinase phosphorylation sites (TEY, residues 174-176) in front of the YRAPE domain (Nishida and Gotoh 1993).

To demonstrate that $P M K 1$ encodes a protein kinase, we constructed glutathione $S$-transferase (GST)-Pmk1 fusions and expressed them in Escherichia coli. A GSTPmkl fusion protein of the expected size of $65 \mathrm{kD}$ was produced and purified with glutathione-agarose beads. In protein kinase assays, the purified GST-Pmk1 fusion protein underwent autophosphorylation and could phosphorylate myelin basic protein (MBP, $20 \mathrm{kD}$ ) (Fig. 2C). But the extent of the GST-Pmk1 autophosphorylation and MBP phosphorylation was lower than that of the positive control, mouse GST-Erk1. We conclude that $P M K 1$ encodes a functional protein kinase.

\section{PMK1 functions in yeast}

The degree of relatedness of PMK1 to the yeast FUS3/ KSS1 genes prompted us to test whether PMK1 could function in the yeast pheromone signaling pathway as a MAP kinase. We placed the PMK1 coding region under the control of the yeast GAL1 promoter in the plasmid pYES2, and introduced the resulting construct pYESA3 into yeast strain yDM300 deleted for the FUS3/KSS1 kinases. The resulting $\mathrm{Ura}^{+}$transformants (relevant genotype, URA3 trp1 $\triangle 1$ ) containing PMK1 were mated to CG219 (relevant genotype, ura3-52 $\mathrm{TRP1}^{+}$) and tested for diploid formation (see Materials and Methods). Without mating, all pYESA3 transformants and control strains grew on YPD but not on SD-Ura-Trp medium (Fig. 3A). Diploids were only observed on SD-Ura-Trp after mating with CG219 on medium containing galactose (Fig. 3A). We conclude that PMK1 can rescue the mating defect of the yeast fus3 kss1 double mutant.

The mating efficiency of yeast strains expressing PMK1 is lower than control strains expressing KSS1 (Fig. 3A). To confirm that these colonies arose as a result of mating, we tested 20 random putative diploid colonies from these matings by yeast mating-type PCR (Huxley et al. 1990) and found that all twenty were true $M A T \mathbf{a} / \alpha$ diploids and contained the pYESA3 plasmid (Fig. 3B). Besides mating, yDM300 transformants (MATa) expressing $P M K 1$ were capable of forming mating projections in the presence of the yeast pheromone $\alpha$-factor (data not shown). 
A

Figure 2. Nucleotide and protein sequences of the PMK1 gene and protein $\mathrm{ki}$ nase activity of GST-Pmk1 fusion protein (A) Nucleotide sequence and deduced amino acid sequence of the PMK1 gene. The lowercase letters represent two intron regions. Amino acid residues are numbered on the left. The different $5^{\prime}$ and $3^{\prime}$ ends of the five $P M K 1$ cDNA clones (A1-A5) are labeled on top. The GenBank accession no. for the PMK1 sequence is U70134. $(B)$ Sequence alignment of $P M K 1$ with $S$. cerevisiae FUS3 and KSS1 and human ERK1. Identical residues are shown in black boxes. Similar residues are shaded. The 11 protein kinase subdomains are labeled on top in roman numerals (Hanks et al. 1988). The tyrosine and threonine residues, two putative phosphorylation sites for MAP kinase kinase, are identified by asterisks. $(C)$ Kinase activity of GST-Pmk1 fusion proteins. (L) Products of autophosphorylation assay separated on $10 \%$ SDS-PAGE gel. (R) Products of MBP phosphorylation assay separated on $15 \%$ SDS-PAGE gel. Lanes $1,2,3$, and 4 are mouse GST-Erk1, boiled mouse GST-Erk1, GST-Pmk1, and boiled GST-Pmkl, respectively. In each lane 0.9 $\mu \mathrm{g}$ of mouse GST-Erkl or $3.5 \mu \mathrm{g}$ of GSTPmk fusion protein was loaded.

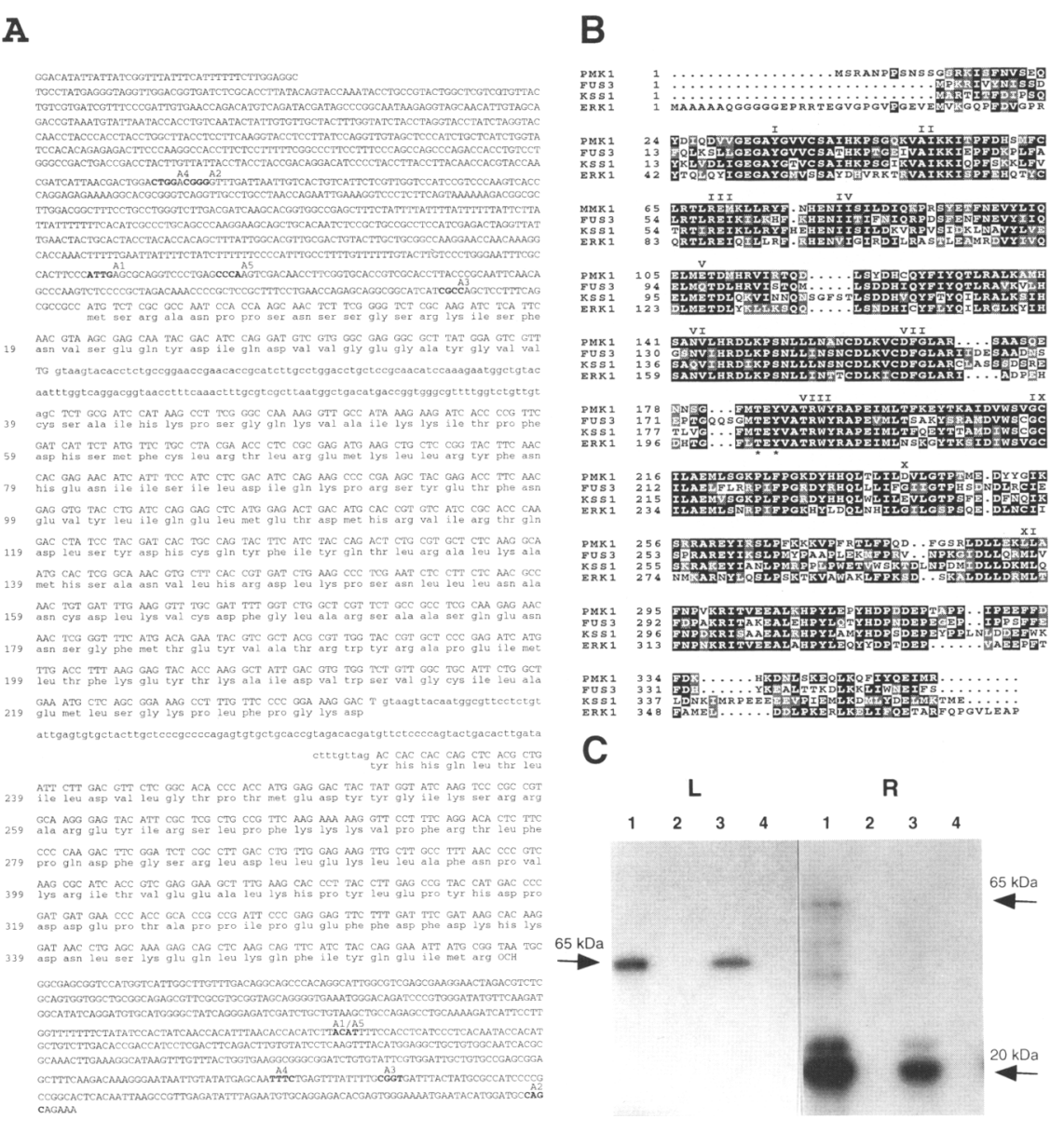

PMKl is essential for pathogenicity and invasive growth

Molecular genetic techniques can be used to introduce recombinant DNA molecules into $M$. grisea. During transformation DNA integrates by both homologous and apparent nonhomologous, ectopic integration (Talbot et al. 1993). We used genomic clones from the PMK1 locus to construct a gene-replacement vector (pSSE3; Fig. 4A) to precisely delete $P M K 1$ and replace it with a gene encoding hygromycin phosphotransferase $(H P H)$. pSSE3 DNA was linearized with $\mathrm{BamHI}$ and transformed into wild-type strain Guyl1 and transformants were selected on hygromycin containing medium. A spore PCR assay (Xu and Hamer 1995) identified 11 out of 104 hygromycin resistant (hygR) transformants that had the PMK1 gene replaced with $H P H$ (data not shown). PMK1 knockout transformants were confirmed to have the correct gene replacement events by Southern blot analysis (Fig. 4B). All hygromycin-resistant transformants examined contained the 1.4-kb Sall fragment encoding the $H P H$ gene. Hybridization analysis with the $\mathrm{Sacl} 2.5-\mathrm{kb}$ fragment from pSC2.5 (Fig. 4A) showed that the wild-type strain Guy11 and transformant nn90 contained the PMK1 4.5-kb Sall fragment. Transformant nn90 also contained a $2.7-\mathrm{kb}$ Sall fragment diagnostic for the gene replacement cassette. We conclude that nn90 contains the gene disruption cassette integrated elsewhere in the genome. Transformants $\mathrm{nn} 26, \mathrm{nn} 78, \mathrm{nn} 92$, and $\mathrm{nn} 95$ do not contain the 4.5-kb Sall fragment from PMK1, but contain the $2.7-\mathrm{kb}$ Sall fragment indicative of the gene replacement event. Additional restriction digests confirmed the correct gene replacement event had occurred in all $11 P M K 1$ knockout transformants /data not shown).

Infection assays on rice cultivar $\mathrm{CO} 39$ were used to test the pathogenicity of pmk1 mutants. Conidial suspensions from each of the $11 \mathrm{pmk} 1$ mutants and control strains were sprayed onto 2-week-old CO39 rice seedlings, and inoculated seedlings were incubated for 5-6 days prior to scoring disease symptoms. Both wild-type strain Guyll and the ectopic insertion transformant nn90 formed numerous lesions over the entire inoculated plant area. Several days later infected leaves from these plants wilted and died. In contrast, the pmk 1 deletion mutant $\mathrm{nn} 78$ failed to produce any lesions on susceptible rice plants (Fig. 5A). Identical results were obtained for all 11 pmk1 strains. Microscopic examination of the infected leaves revealed a complete absence of hypersensitive lesions, suggesting that pmk1 strains failed to penetrate the plant cuticle.

M. grisea strain Guyll can also cause lesions on barley 
$\mathbf{A}$
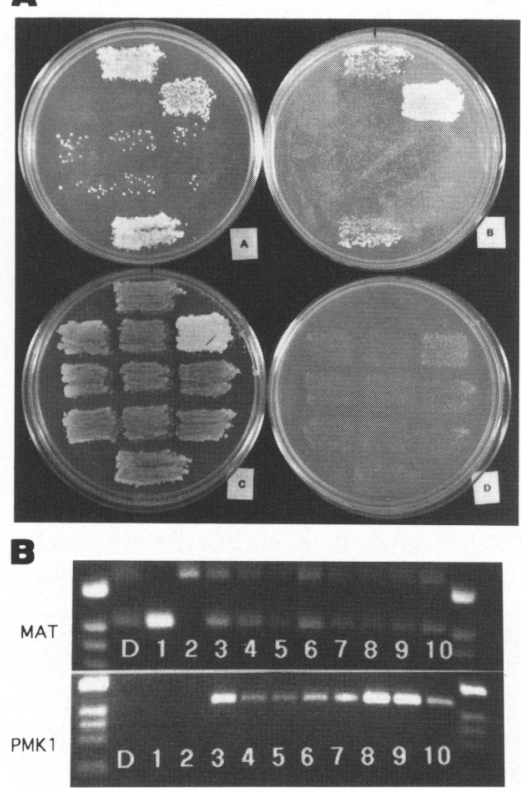

Figure 3. $P M K 1$ functions as a MAP kinase in yeast. $|A|$ Yeast mating assay. Diploid formation was examined by replica plating to SD-Ura-Trp medium following mating with CG219

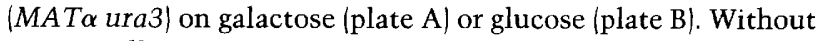
mating, all strains grew on YPD (plate C), but not on SD-UraTrp (plate D). Top and bottom patches were fus $3 \mathrm{kss} 1$ double mutant yDM300 transformed with pBC50 (carrying yeast KSS1 gene). From left to right, patches in the second row are yPH499 (MATa trp1 ura3), yDM300 (MATa fus3 kss1 trp1 ura3) and XK1-25 (MATa trp1). All six patches in the third and fourth rows were yDM300 transformants (MATa fus3 kss1 trp1) containing pYESA3 (PMK1 gene controlled by GAL1 promoter). (B) Test of the diploid colonies by PCR with yeast $M A T$ primers (top) and PMK1 primers (bottom). Lane $D$ is a diploid $(M A T \mathbf{a} / \alpha)$ control strain formed by XK15 and CG219. Lanes 1 and 2 are haploid strains XK15 (MATa) and CG219 (MAT $\alpha)$. Lanes 3-10 are random putative diploids formed by mating CG219 with pYESA3 transformants on galactose medium. Lanes on both sides are the 1-kb ladder molecular weight marker (BRL).

cultivar Golden Promise. Inoculation assays on barley gave identical results to rice infection assays /data not shown), demonstrating that the failure of pmk1 strains to infect is not host-specific. To confirm that this phenotype was due to the deletion of the PMK1 gene, we crossed pmk1 mutant nn 78 with a wild-type tester strain 4136-4-3 and isolated 45 random ascospore progeny. The progeny segregated $\sim 1: 1$ for hygromycin resistance and there was complete cosegregation of the nonpathogenicity phenotype with the hygR phenotype /data not shown).

The ability of $M$. grisea strains to cause disease and grow invasively can be assessed independently of penetration by inoculating through wounding (Chumley and Valent 1990). To test the role of PMK1 in invasive growth in plants, mutant and control strains were injected into leaf sheaths of susceptible rice seedlings (see
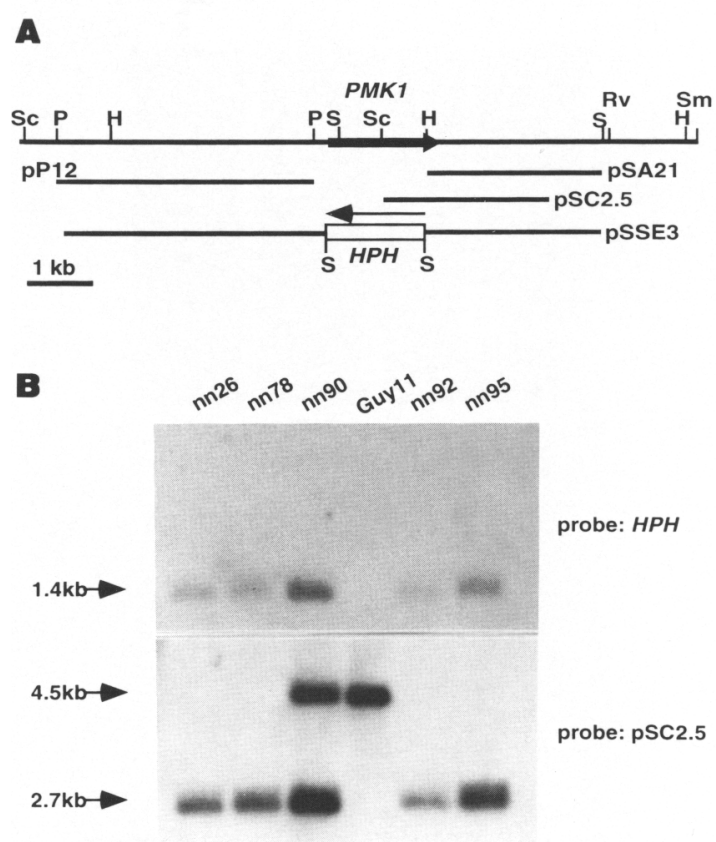

Figure 4. $P M K 1$ gene replacement vector and transformants. (A) Physical map of the PMK1 genomic region and the gene replacement vector pSSE3. The restriction enzymes are: (Sc) SacI; (H) HindIII; (P) PstI; (Rv) EcoRV; (S) SalI; (Sm) SmaI. The arrows indicate the directions of $P M K 1$ and $H P H$ genes. $(B)$ Southern blot of wild-type (Guy11), ectopic integration transformant (nn90), and PMK1 gene replacement transformants (nn26, nn78, nn92, and nn95). All DNA samples were digested with Sall. The blot was probed with the HPH gene (top), then stripped and reprobed with the $S a c I 2.5-\mathrm{kb}$ fragment from pSC2.5.

Materials and Methods). Injections with Guyll caused lesions in and around the wound sites caused by the injection (Fig. 5B). Similar results were obtained for strains containing ectopic integration events /data not shown). Injections with a $0.2 \%$ gelatin solution resulted in predominantly white wound sites with little or no plant cell necrosis (Fig. 5B). Injections with pmk1 mutants resulted in small areas of necrosis at the site of injection but a complete absence of spreading lesions outside of the wound sites (Fig. 5B). To test whether pmk1 strains were still alive in these wound sites, we excised 50 wound sites from plants injected with either wild-type or mutant strains. Excised leaf sections were placed on water agar plates and incubated with direct light and high humidity for 2-3 days. Under these conditions, wound sites inoculated with wild-type strain Guy11 produced abundant conidia and mycelia (Fig. 5C). Buf $^{-}$mutants of $M$. grisea, which can not penetrate healthy leaf surfaces because of a defective melanin wall layer in the appressoria (Chumley and Valent 1990), also served as a positive control. When wound sites inoculated with Buf ${ }^{-}$mutants were excised and incubated under the same conditions, mycelial growth and conidiation were observed. However, no mycelia or conidia ever 


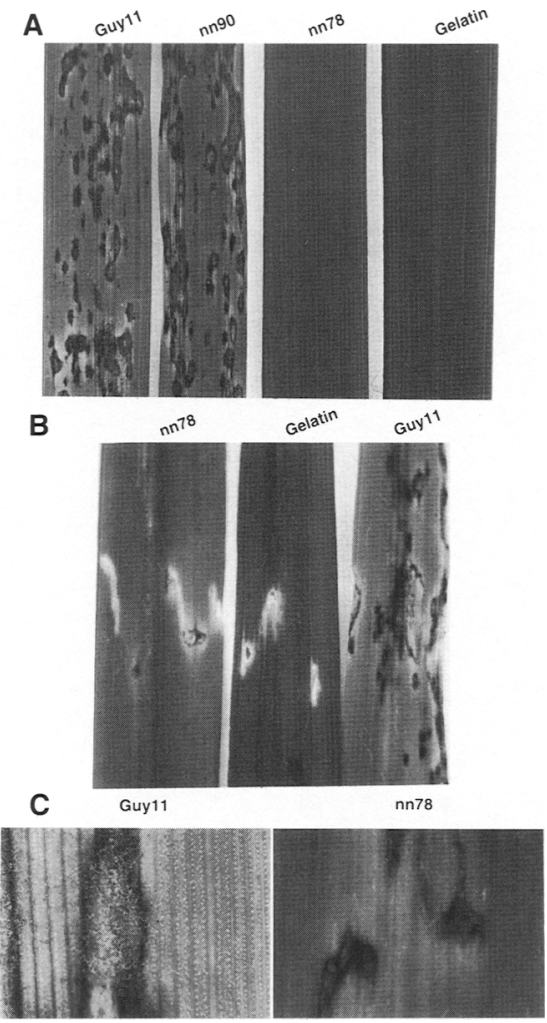

Figure 5. $P M K 1$ is required for pathogenicity on rice. $(A)$ Seedlings from rice cultivar $\mathrm{CO} 39$ were spray-inoculated with either wild-type (Guy11), ectopic integration transformant (nn90), pmk1 (nn78), or $0.25 \%$ gelatin solution (Gelatin). Typical leaves are shown following 1 week of symptom development. $(B)$ From left to right are leaves from $\mathrm{CO} 39$ plants wound-inoculated with nn78, gelatin solution, and Guyl1. $(C)$ Lesions from woundinoculated rice plants were incubated with light and humidity for 2 days. Sites inoculated with Guyll (left) produced a gray mycelium, conidiophores and conidia on the leaf surface, but sites inoculated with nn78 (right\} did not have any fungal growth or sporulation.

emerged from wounds inoculated with pmk1 mutants (Fig. 5C). We conclude that PMK1 is required for both penetration and invasive growth or viability in plants.
PMKl is required for infection-related morphogenesis

pmk1 strains could fail to penetrate plant leaves as a result of an inability of spores to germinate, to form appressoria, and/or to generate sufficient turgor pressure to penetrate the plant cuticle. Infection-related morphogenesis can be followed by observing germinating conidia in distilled water on hydrophobic Teflon membranes (Hamer et al. 1988). All pmk1 strains germinated efficiently but failed to form appressoria (Table 1). To define this defect more precisely we followed infection-related morphogenesis in wild-type strain Guyl1 and pmk1 strain nn78. At various time intervals, cells germinating on membranes were fixed and stained with a combination of Calcofluor (to stain cell walls) and Hoechst 33258 (to stain nuclei) and observed with fluorescence and/or differential interference contrast microscopy (Fig. 6).

Both Guy 11 and nn78 produced abundant conidia with a characteristic pyriform shape containing three uninucleate cells delimited by two septa (Fig. 6A; $0.5 \mathrm{hr}$ ). The tapered tip of the conidia releases a Calcofluor bright staining adhesive called spore tip mucilage (STM; Hamer et al. 1988) that attaches the conidium to hydrophobic surfaces (Fig. 6A). Wild-type conidia germinated by producing a thin germ tube within $30 \mathrm{~min}$. By $2 \mathrm{hr}, \sim 20 \%$ of the germ tubes underwent tip swelling and differentiation and by $8 \mathrm{hr}$ the majority of the wild-type cells had formed appressoria (Table 1). In wild-type cells appressorium formation is accompanied by a single round of nuclear division followed by the formation of a septum at the base of the appressorium (Fig. 6A; Bourett and Howard 1990). By $24 \mathrm{hr}>90 \%$ of the germinated wildtype conidia had formed mature appressoria. Following appressorium formation, no further tip growth or nuclear division occurs, and the germ tube and spore cells become vacuolated and collapsed (Fig. 6A, 24 hr; Bourett and Howard 1990; Howard 1994).

In pmk1 mutant nn78, conidia attached to the surface and started germination within $30 \mathrm{~min}$. Less than $5 \%$ of the germ-tube tips started swelling or differentiation at 2 hr (Fig. 6A, Table 1). However, by $8 \mathrm{hr}$ a majority of the germ-tube tips were hooking, swelling, and becoming highly deformed (Fig. 6A, Table 1). By $24 \mathrm{hr},>90 \%$ of the germ tubes underwent hooking and subapical swelling

Table 1. Thigmotropic response in wild type strain Guy11 and pmk1 mutant nn78

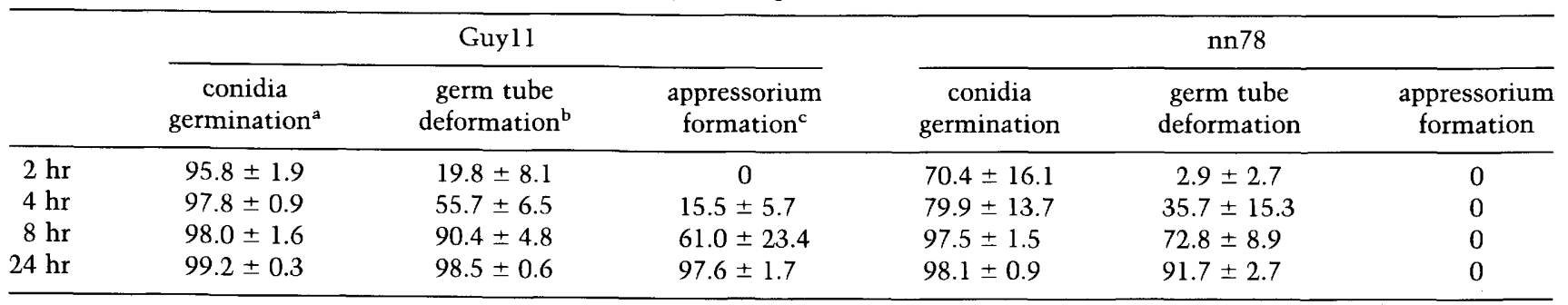

${ }^{a}$ For each time point, at least 100 conidia were examined to calculate the percentage of germination. The mean and standard deviation were calculated from at least three independent trials.

${ }^{b}$ Germ tube deformation: the percentage of germ tubes with hooking and subapical swollen structures (Fig. 4A).

'Appressorium formation: the percentage of all germ tubes that formed appressoria. Of the nn 78 germ tubes examined at 24 and 48 hours $(n>1000)$, no appressoria were observed. 
A
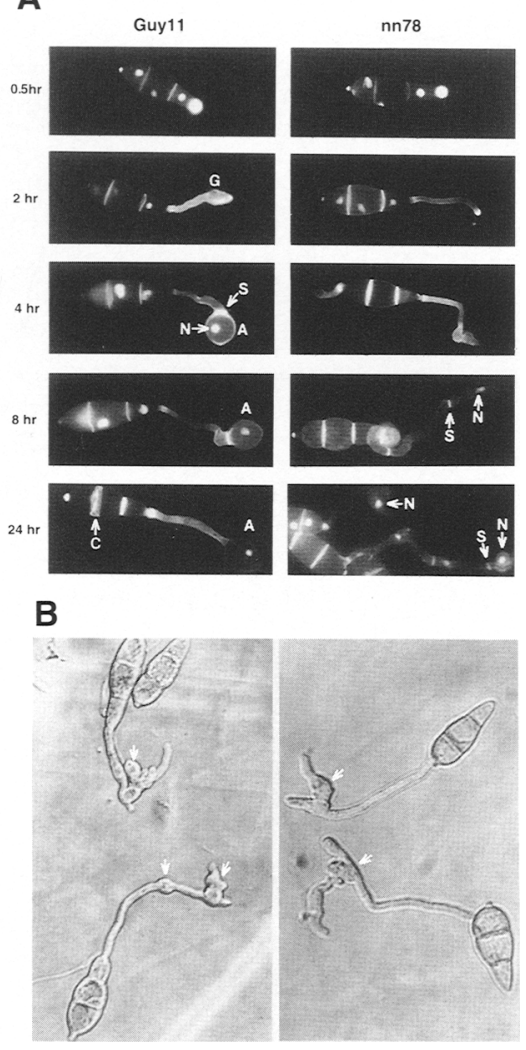

Figure 6. $P M K 1$ is required for infection structure formation. (A) Conidia germinated on Teflon membrane at $25^{\circ} \mathrm{C}$ were removed at indicated times and stained with Calcofluor to visualize cell walls and Hoechst 33258 to visualize nuclei $(560 \times$ magnification). The panels depict typical stages of infection-related morphogenesis in Guyll (wild-type) or nn78 (pmk1). Nuclear staining in the three celled conidia from both wild-type and mutants gave variable staining after $4 \mathrm{hr}$. At 24 hr, wild-type conidial cells were vacuolated. (A) Appressorium; (G) germ tube; (N) nucleus; (S) septum; (C) collapsed conidial cells. (B) Subapical swollen structures in strain nn78. Both photos are conidia germinated on Teflon membranes and photographed after $24 \mathrm{hr}$ with Nomarski microscopy (560× magnification). Arrows indicate the subapical swollen structures.

(Fig. 6B), but no appressoria were formed (Table 1). Even after $48 \mathrm{hr}$, none of the nn78 germ tubes $(>1000)$ examined formed any appressoria. The subapical swollen structures were much smaller than appressoria and lacked thickened melanized cell walls. These phenotypes suggest that these structures arose as a consequence of continuous growth of the swelling germ tube and a failure to form new cell wall layers or generate high levels of turgor pressure. Some of these swollen structures contained nuclei (Fig. 6A, 24 hr). However, unlike wild-type strains, nn78 strains often underwent two or more rounds of nuclear division and formed septa along the germ tubes and near the subapical swollen structures (Fig. 6A). Furthermore, after $24 \mathrm{hr}$, few of the nn 78 spores underwent collapse and vacuolization. We conclude that pmk1 strains fail to infect plants because of an inability to complete infection-related morphogenesis.

\section{PMK1 may function downstream of a cAMP-dependent signal}

Although hydrophobic surfaces are favored for appressorium formation in $M$. grisea, high extracellular concentrations of cAMP (10 mM) will induce appressorium formation on noninducible hydrophilic surfaces such as glass (Lee and Dean 1993a; Fig. 7). Similar results have been obtained with other surface sensing pathogenic fungi, suggesting that a conserved cAMP signaling pathway may be involved in thigmotropic sensing (Epstein et al. 1989). We tested the effects of hydrophilic surfaces and exogenous $10 \mathrm{mM}$ cAMP on appressorium formation of pmk1 mutants. On a hydrophilic glass surface in the absence of cAMP, the majority of wild-type and pmk1 conidia produced undifferentiated hyphae. These results strongly suggest that PMK1 is not involved in thigmotropic sensing per se. The addition of exogenous cAMP did not rescue the appressorium defective phenotype in pmk1 strains; however, it did elevate the level of germtube hooking and swelling, on a hydrophilic glass surface (Fig. 7). The internal swollen structures, germ tube tip swelling, and deformation closely resembled the behavior of pmk1 strains on Teflon membranes (see Fig. 6A). Thus pmk1 mutants can respond to both thigmotropic surface signals and a cAMP inducible signal; however, pmk1 mutants fail to complete the formation of mature appressoria.

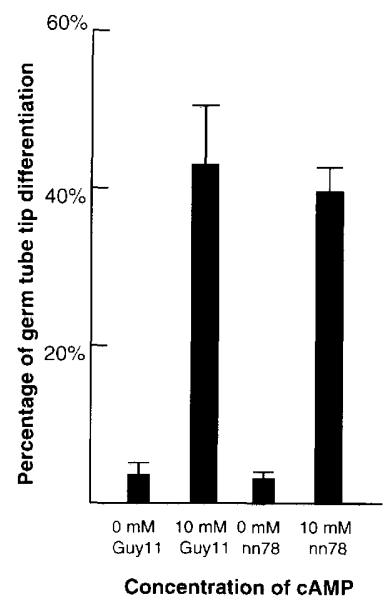

Figure 7. pmk1 mutants retain cAMP responsiveness. Wildtype Guyll and pmk1 mutant nn78 conidia were germinated on a hydrophilic glass surface in the presence or absence of cAMP. cAMP induced appressorium formation in Guy 11 and germtube swelling and hooking in nn78. Germ-tube tip differentiation was counted as the percentage of appressorium formation in Guy11 or germ-tube swelling and hooking in nn78 on germinated conidia. Standard deviations of five assays are indicated above the bars. 


\section{PMKl is dispensable for growth in culture}

M. grisea is heterothallic and contains a single matingtype locus (MAT1) with two alleles. Although little is known about mating processes such as protoperithecia formation and fertilization in $M$. grisea, all $11 \mathrm{pmk} 1$ knockout mutants (mating type MAT1-2) were able to mate with 4136-4-3 (MAT1-1) and produce fertile perithecia as efficiently as the ectopic transformants. Among the 31 asci examined from one cross, 28 had more than four ascospores. Thus pmk1 strains appear to have no defect in ascospore delimitation.

To identify other roles for $P M K 1$ we searched for possible defects in pmk1 mutants during vegetative growth in culture. Growth rates were very similar, about $3 \mathrm{~mm}$ per day for strains nn78, Guyl1, and nn90 in race tube culture, and hyphal morphologies and the extent of asexual spore production (conidiation) were indistinguishable (data not shown). We did not detect differential sensitivities to growth at high temperature or in complete medium containing $0.7 \mathrm{M} \mathrm{KCl}$. We conclude that PMK1, just like FUS3 and KSS1 in yeast, does not play any role in vegetative growth.

\section{Discussion}

In many fungi-plant interactions, physical properties of the plant surface including surface hydrophobicity and architecture are sufficient to elicit fungal recognition (Hoch and Staples 1991). This recognition involves a series of morphological changes in the germinating fungal spore that ultimately results in the attachment and penetration of the plant surface. The invading fungal pathogen must then overcome the host defense system and proliferate by using the host as a substrate. PMK1, a MAP kinase gene isolated from the rice blast fungus, is specifically involved in signaling infection structure formation in response to a thigmotropic surface signal and is also necessary for invasive growth or viability in rice plants.

\section{A MAP kinase pathway for appressorium formation and pathogenic growth}

PMK1 encodes a polypeptide with subdomains characteristic of MAP kinases (Nishida and Gotoh 1993). PMK1 is most similar to yeast FUS3 and KSS1 MAP kinase genes, and a GST-Pmkl fusion protein demonstrated protein kinase activity in vitro. Induced expression of $P M K 1$ was capable of rescuing the phenotype of yeast fus3 kss1 double mutants in mating assays. In order to function in place of the Fus3/Kss1 kinases in yeast, Pmkl must be activated by the yeast MAP kinase kinase (MAPKK) Ste7 (Errede et al. 1993). We have not identified other components of the $M$. grisea MAP kinase pathway, but the function of Pmkl in yeast suggests that Pmk1 may be part of a MAP kinase module (Herskowitz 1995) in M. grisea. Although the upstream kinases in the Pmkl pathway await identification, a distinct possibility is that they will be related to the Ste7 and Ste11 (Rhodes et al. 1990) kinases from yeast.

Our studies show that Pmkl is a MAP kinase that appears to be specific for signaling events in fungal pathogenesis. Pmk1 can function in the yeast pheromone MAP kinase pathway but is dispensable for vegetative growth and sexual reproduction in $M$. grisea. Importantly, a deletion of PMK1 completely abolishes pathogenicity toward rice by blocking the morphogenesis of infection structures important for plant penetration and by abolishing the ability of $M$. grisea to grow and complete its disease cycle in rice plants. In the distantly related corn smut pathogen, Ustilago maydis, pheromones and their receptors are required for cell fusion and the formation of a filamentous dikaryon (Banuett 1992; Hartmann et al. 1996). The filamentous dikaryon is pathogenic to maize plants where it grows and produces tumors. A $U$. maydis gene related to the yeast MAPKK $S T E 7$, called Fuz7, is required for the pheromone-induced formation of the filamentous dikaryon and tumor formation in maize plants (Banuett and Herskowitz 1994). We speculate that although pathogenic processes in fungi may be distinct, a conserved signaling pathway involving an Pmk1-like MAP kinase and upstream kinases related to the yeast Ste7 and Stell kinases may be utilized widely by pathogenic fungi to signal the presence of a suitable host and induce fungal growth processes necessary for pathogenesis.

Like many other fungi where mating is controlled by mating-type genes, the mating response in $M$. grisea is likely to involve mating pheromones, receptors, and a signal transduction pathway (Staben 1995). Thus one explanation for the specific role of Pmkl in pathogenesis is that a related MAP kinase may exist that regulates the mating response in $M$. grisea (analogous to yeast FUS3/ KSS1). Our screen thus far has failed to identify such a MAP kinase, even though genes homologous to the yeast SLT2 (Lee et al. 1993) and HOG1 (Brewster et al. 1993) MAP kinases have been recovered (J.R. $\mathrm{Xu}$ and J.E. Hamer, unpubl.). Interestingly, mating and sexual reproduction is dispensable for $M$. grisea in nature (Leung and Williams 1985; Levy et al. 1991; Notteghem and Silue 1991). The hypothesis that the fungal mating response and pathogenesis signal transduction pathways share components may help to explain why some pathogenic fungi like $M$. grisea retain a vestigial sexual cycle, even though its sexual cycle is dispensable for pathogenesis.

\section{cAMP and MAP kinase pathways for pathogenesis in M. grisea}

cAMP signaling appears to be essential for appressorium formation in $M$. grisea. Exogenous cAMP stimulates appressorium formation on hydrophilic surfaces, and a deletion of the single-copy CPKA gene results in a delay in appressorium (Mitchell and Dean 1995; our unpubl. data). Although pmk1 strains fail to form appressoria on Teflon membranes, they are able to undergo germ-tube swelling and hooking in response to exogenous cAMP on hydrophilic surfaces. The phenotypes of pmk1 mutants 
suggest that Pmk1 may be necessary for arresting nuclear division and growth following appressorium formation. PMK1 may also be necessary for signaling downstream events such as the formation of new wall layers or turgor pressure in the appressorium. These findings suggest that Pmkl kinase may act downstream of a cAMP-dependent signal, and it is possible that high intracellular levels of cAMP may activate the PMK1 MAP kinase pathway. Alternatively, related or different thigmotropic signaling events may activate the CPKA and $P M K 1$ kinases independently. Ultimately, coordination between these signaling pathways is essential for pathogenesis.

Appressorium formation is a complex morphological process involving changes in the fungal cytoskeleton (Kwon et al. 1991) and the synthesis of new cell wall layers (Bourett and Howard 1990). In M. grisea, the appressorium is also a terminally differentiated cell type, and further cell growth and nuclear division is inhibited unless the appressorium can penetrate the underlying surface (Bourett and Howard 1990; our unpubl. data). In order to penetrate the underlying plant surface, the appressorium generates turgor pressure most likely through the synthesis of a compatible solute such as glycerol from stored carbohydrate reserves (Howard 1994). These biochemical changes suggest possible roles for the protein kinase signaling pathways necessary for pathogenesis in M. grisea (Fig. 8). CPKA may be required for the mobilization of stored glycogen and trehalose re-

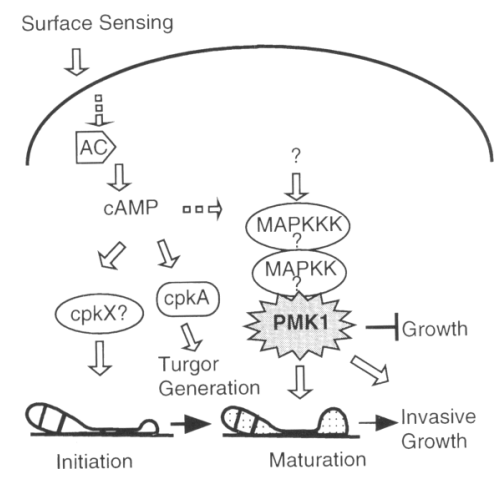

Figure 8. A model for MAP kinase and cAMP signaling for plant pathogenesis by the rice blast fungus. Contact with an inducible hydrophobic surface increases intracellular levels of cAMP presumably by activation of adenylate cyclase (AC). cAMP activates the cAMP-dependent protein kinase(s) that may promote the mobilization of storage carbohydrates and turgor generation in appressoria. Because $c p k A$ mutants are still able to form appressoria and are responsive to exogenous CAMP, additional catalytic subunit(s) of cAMP dependent protein kinase $(c p k X)$ may exist and stimulate the initiation of appressorium formation. Later events in appressorium formation include arrest of germ-tube tip growth, synthesis of specific cellwall layers, and turgor pressure generation. These later appressorium maturation events are stimulated by the MAP kinase $P M K 1$, presumably through a MAP kinase module that may respond to the cAMP signal. Pmkl signaling is also necessary for invasive growth in rice plants. serves in the spore (Howard et al. 1991; Howard 1994). cAMP and protein kinase A (PKA) in yeast (van der Plaat 1974; Thevelein 1994) are known to play critical roles in mobilization of stored carbohydrates. Downstream of $C P K A$, we speculated that Pmk1 may signal a growth arrest and the activation of appressorial specific genes for new cell wall biosynthesis and turgor generation (Fig. 8). In conclusion, infection structure formation in $M$. grisea requires cooperative signaling between cAMP and MAP kinase-dependent pathways. We speculate that this cooperative signaling may be a common feature in fungal pathogens that form infection structures.

Finally, $P M K 1$ is also necessary for invasive fungal growth or viability in rice plants (Fig. 8). Although little is known about this growth stage in $M$. grisea, genes expressed during pathogenic growth and putative regulators of these genes have been identified in $M$. grisea (Talbot et al. 1993; Lau and Hamer 1996). The specific roles of PMK1 in infection-related morphogenesis and invasive growth suggest that conserved fungal signal transduction pathways will present useful targets for the future design of antifungal molecules.

\section{Materials and methods}

PCR cloning of the PMKl gene

Three degenerate primers, GG(C/T)TT(A/C/G/T)A/A/C/G/ T)(A/G)TC(A/C/G/T)C(G/T)(A/G)TG (MEK3), GT(A/C/G/ $\mathrm{T}) \mathrm{GC}(\mathrm{A} / \mathrm{C} / \mathrm{G} / \mathrm{T}) \mathrm{AT}(\mathrm{A} / \mathrm{G}) \mathrm{AA}(\mathrm{A} / \mathrm{G}) \mathrm{AA} / \mathrm{A} / \mathrm{G}) \mathrm{AT}$ (MAK2), and $\mathrm{TC}(\mathrm{A} / \mathrm{C} / \mathrm{G} / \mathrm{T}) \mathrm{GG}(\mathrm{A} / \mathrm{C} / \mathrm{G} / \mathrm{T}) \mathrm{GC}(\mathrm{A} / \mathrm{C} / \mathrm{G} / \mathrm{T}) \mathrm{C}(\mathrm{G} / \mathrm{T})(\mathrm{A} / \mathrm{G}) \mathrm{TA} / \mathrm{A} /$ $\mathrm{C} / \mathrm{G})(\mathrm{C} / \mathrm{T} / \mathrm{A}$ (MAK4), were designed according to the conserved amino acid residues in yeast MAP kinases FUS3, KSS1, HOG1, and $S M K 1$ (Krisak et al. 1994). The primary PCR reaction was conducted with primers MAK2 and MAK4 using first-strand cDNA generated from polyadenylated RNA prepared from nitrogen-starved mycelial culture. Nested PCR was performed with primers MAK2 and MEK3 using the diluted primary PCR product. The PCR reaction involved 30 cycles $\left(1 \mathrm{~min}, 94^{\circ} \mathrm{C} ; 2\right.$ $\min , 52^{\circ} \mathrm{C} ; 2 \mathrm{~min}, 72^{\circ} \mathrm{C}$ ) followed by $5 \mathrm{~min}$ at $72^{\circ} \mathrm{C}$. The nested PCR products were cloned in pGEM-T (Promega) and sequenced. PCR clones having homology with yeast FUS3 and KSS1 were used to screen cDNA clones from a $\lambda$ ZAPII (Stratagene) cDNA library constructed with RNA isolated from nitrogen-starved Guy11 mycelial culture and genomic clones from a Guy11 1 GEM-11 genomic library (Talbot et al. 1993).

\section{DNA/RNA manipulations and sequence analysis}

Total RNA was prepared from fungal mycelia using the method described by Timberlake (1980). Polyadenylated RNA was isolated with the PolyATract mRNA isolation kit (Promega). Standard molecular biology procedures were followed for the first strand cDNA and DNA manipulations (Sambrook et al. 1989). Fungal DNA was extracted with the CTAB protocol (Xu and Leslie 1996). DNA samples were sequenced using Sequenase V2.0 DNA sequencing kit (U.S. Biochemicals). Homolog search of DNA/protein sequence data bases was performed with the BLAST programs (Altschul et al. 1990). Amino acid sequence comparison and alignments were made with the BESTFIT, PILEUP, and BOXSHADE programs in GCG software package (University of Wisconsin). 
Magnaporthe culture, transformation, and crossing

Wild-type strains Guy11, 4136-4-3, and 4375-R-26 were cultured at $25^{\circ} \mathrm{C}$ on oatmeal plates under fluorescent light for conidiation (Romao and Hamer 1992). Growth conditions for nitrogen starvation and normal complete media cultures were as described previously (Talbot et al. 1993). Guyl1 protoplast preparation and transformation was performed as described (Sweigard et al. 1992). Putative hygromycin resistant transformants were confirmed by spore-PCR (Xu and Hamer 1995) with $H P H$ primers GTCCGTCAGGACATTGTT (H850) and ACTCACCGCGACGTCTGT (H852). All true transformants were purified by monoconidial isolation. Genetic crosses and progeny isolations were performed as described (Crawford et al. 1986). Asci were stained with $0.1 \%$ lacto-phenol cotton blue to examine ascospores.

\section{Construction of the gene replacement vector pSSE3}

Plasmid pSA21 was constructed by cloning the SalI-HindIII 2.7kb fragment ( 3 ' end of $P M K 1$ ) in pBluescript $\mathrm{KS}+$ (Stratagene) as pSA21. The PstI 4.5-kb fragment (upstream of PMK1) was cloned in $\mathrm{pBC}$ SK - (Stratagene) as $\mathrm{pP12}$. This $4.5-\mathrm{kb}$ insert fragment was released from $\mathrm{pP} 12$ with EcoRI and SacI, and then ligated into pSA21 (Fig. 4A) to make plasmid pSE1. The gene replacement vector pSSE3 was constructed by inserting the modified hygromycin phosphor transferase $(H P H)$ gene (Carroll et al. 1994) into the EcoRI site on pSE1 to create pSSE3. pSSE3 was linearized with BamHI and transformed into Guyll.

\section{Rice infection assays}

Conidia were collected from 10- to 14-day-old oatmeal agar cultures and resuspended to $5 \times 10^{5}$ conidia per $\mathrm{ml}$ in $0.25 \%$ gelatin. Two-week-old seedlings of rice cultivar CO39 or barley cultivar Golden Promise were used for infection assays. Plant incubation and spray inoculation were as described (Valent et al. 1991). Lesions were examined 5-6 days after inoculation. For injection inoculation, conidial suspensions were injected into leaf sheaths of rice seedlings with $26 \mathrm{G}_{1 / 2}$ needles. Lesion formation and necrosis around the inoculation sites were examined when the injection-wounded leaves unfolded 4-7 days after injection. Wound inoculation sites were excised, surface-sterilized with $70 \%$ ethanol for $1 \mathrm{~min}$ and incubated on $2 \%$ water agar with fluorescent light and high humidity for sporulation assays.

\section{Assays for infection structure formation}

Conidia were prepared and assayed for appressoria formation as described previously (Hamer et al. 1988). After incubation at $25^{\circ} \mathrm{C}$ for specific times, germinating conidia were stained with $10 \mu \mathrm{g} / \mathrm{ml}$ Calcofluor and $100 \mathrm{ng} / \mathrm{ml}$ Hoechst 33258 as described (Harris et al. 1994) and examined by epifluorescence microscopy. Photographs were taken with Kodak Technical-Pan films. Conidia were resuspended in $10 \mathrm{mM}$ cAMP and tested for cAMP responsiveness as described (Lee and Dean 1993a).

\section{GST-Pmk1 fusion protein and phosphorylation assay}

The full-length $P M K 1$ open reading frame was amplified with primers MK15 (GGAATTCTCATGTCTCGCGCCAATCCA) and MMK3X (CCGCTCGAGCCGCATAATTTCCTGGTAGAT). The PCR product was digested with EcoRI and XhoI, and cloned in-frame into pGEX-KG (Guan and Dixon 1991). Expression and purification of GST-Pmk1 fusion proteins followed the techniques described by Smith and Johnson (1988). The mouse GST-Erk1 fusion protein was a gift of Curtis Ashendel (Purdue University|. Protein kinase assays were performed as described by Crews et al. (1991). Boiled GST-Pmk1 and GST-Erkl proteins were used as negative controls. Autophosphorylation and myelin basic protein phosphorylation were examined by autoradiography after electrophoresis on $10 \%$ and $15 \%$ SDS-PAGE gel.

\section{Yeast mating assay}

Yeast strains XK1-25 (MATa trp1-289) and CG219 (MAT $\alpha$ ura352) were obtained from Dr. Gunter Kohlhaw (Purdue Univer-

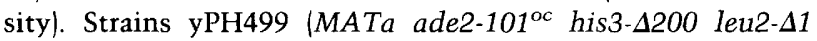
lys2-801 ${ }^{a m}$ trp1 11 ura3-52) and yDM300 (MATa fus3-6::LEU2 kss $1:: H I S 3$ trp1 1 1 ura3-52; Ma et al. 1995) were kindly provided by Dr. Duane Jenness (University of Massachusetts Medical Center). Plasmid pYESA3 with $P M K 1$ under yeast GAL1 promoter control was constructed by cloning the $\mathrm{XhoI}$ and $\mathrm{BamHI}$ fragment of $P M K 1$ cDNA clone A3 into Xhol/BamHI doubledigested pYES2 vector (InVitrogen). pYESA3 was transformed into yDM300 by electroporation, and the resulting Ura ${ }^{+}$transformants were mated with CG219 as described by Sprague (1991). Strains XK1-25 and yDM300 transformed with pBC50 (Courchesne et al. 1989) carrying yeast KSS1 gene behind GAL1 promoter were used as positive controls for mating. The two negative controls were yPH499 $\left(\mathrm{Ura}^{-}\right.$and $\left.\mathrm{Trp}^{-}\right)$and yDM300. The master plate was prepared by applying small patches of pYESA 3 transformants and control strains onto YP-raffinose (YPR) medium ( $1 \%$ yeast extract, $2 \%$ peptone, $2 \%$ raffinose). After overnight incubation at $30^{\circ} \mathrm{C}$, the master plate was replicated onto a YP-galactose (YPG) plate spread with $10^{6}$ CG219 cells, a YP-dextrose (YPD) plate spread with CG219 cells, and a YPD control plate with no CG219 cells. A separate velvet was used for each plate. After overnight incubation at $30^{\circ} \mathrm{C}$, all three plates were replicated onto SD-Ura-Trp and YPD plates. The presence of prototrophic diploids resulting from mating were scored as yeast growth at the position of a patch after 24-hr incubation. The putative diploid colonies were assayed by colony PCR with yeast MAT primers (Huxley et al. 1990) for the presence of MATa and MAT $\alpha$ alleles. The existence of pYESA3 in the diploid colonies were confirmed by PCR with $M K K 1$ primers ACAAGCCAATGACCATG (A5R3) and CCATCGATATCTGGCTGAAATGCTCAGC (MAKF3). The PCR products are $404 \mathrm{bp}$ for $M A T \alpha, 544 \mathrm{bp}$ for $M A T a$, and $460 \mathrm{bp}$ for PMK1.

\section{Acknowledgments}

We thank Drs. Gunter B. Kohlhaw and Duane Jenness for yeast strains, Gee Lau for the cDNA library, Curtis Ashendel for mouse GST-Erk1, and William Courchesne for plasmid pBC50. We also thank Drs. Miriam Hasson, Lisbeth Hamer, and Susan Kaminsky for useful suggestions to the manuscript. This work was supported in part by a National Science Foundation Grant and a Presidential Faculty Fellow Award to J.E.H.

The publication costs of this article were defrayed in part by payment of page charges. This article must therefore be hereby marked "advertisement" in accordance with 18 USC section 1734 solely to indicate this fact.

\section{References}

Altschul, S.F., W. Gish, W. Miller, E.W. Myers, and D.J. Lipman 1990. Basic local alignment search tool. I. Mol. Biol. 215: 403-410. 
Banuett, F. 1992. Ustilago maydis, the delightful blight. Trends Genet. 8: 174-180.

Banuett, F. and I. Herskowitz. 1994. Identification of Fuz7, a Ustilago maydis MEK/MAPKK homolog required for a-locus-dependent and -independent steps in the fungal life cycle. Genes \& Dev. 8: 1367-1378.

Bourett, T.M. and R.J. Howard. 1990. In vitro development of penetration structures in the rice blast fungus Magnaporthe grisea. Can. J. Bot. 68: 329-342.

Brewster, J.L., T.D. Valoir, N.D. Dwyer, E. Winter, and M.C. Gustin. 1993. An Osmosensing signal transduction pathway in yeast. Science 259: $1760-1763$

Carroll, A.N., J.A. Sweigard, and B. Valent. 1994. Improved vectors for selecting resistance to hygromycin. Fungal. Genet. Newsl. 41: 22.

Chumley, F.G. and B. Valent. 1990. Genetic analysis of melanin-deficient, nonpathogenic mutants of Magnaporthe grisea. Mol. Plant-Microbe Interact. 3: 135-143.

Courchesne, W.E., R. Kunisawa, and J. Thorner. 1989. A putative protein kinase overcomes pheromone-induced arrest of cell cycling in Saccharomyces cerevisiae. Cell 58: 11071118.

Crawford, M.S., F.G. Chumley, C.G. Weaver, and B. Valent. 1986. Characterization of the heterokaryotic and vegetative diploid phases of Magnaporthe grisea. Genetics 114: 11111129.

Crews, C.M., A.A. Alessandrini, and R.L. Erikson. 1991. Mouse Erk-1 gene product is a serine/threonine protein kinase that has the potential to phosphorylate tyrosine. Proc. Natl. Acad. Sci. 88: 8845-8849.

Epstein, L., R.C. Staples, and H.C. Hoch. 1989. Cyclic AMP, cyclic GMP, and bean rust Uredospore germlings. Exp. Mycol. 13: 100-104.

Errede, B., A. Gartner, Z. Zhou, K. Nasmyth, and G. Ammerer. 1993. MAP kinase related FUS3 from $S$. cerevisiae is activated by STE7in vitro. Nature 362: 261-264.

Ford, T.L., J.T. Cooley, and P. Christou. 1994. Current status for gene transfer into rice utilizing variety-independent delivery systems. In Rice blast disease (ed. R.S. Zeigler, S.A. Leong, and P.S. Teng), pp. 195-208. CAB International, Wallingford, UK.

Guan, K.L. and J.E. Dixon. 1991. Eukaryotic proteins expressed in Escherichia coli: An improved thrombin cleavage and purification procedure of fusion proteins with glutathione S-transferase. Anal. Biochem. 192: 262-267.

Hamer, J.E., R.J. Howard, F.G. Chumley, and B. Valent. 1988. A mechanism for surface attachment in spores of a plant pathogenic fungi. Science 239: 288-290.

Hanks, S.K., A.M. Quinn, and T. Hunter. 1988. The protein kinase family: Conserved features and deduced phylogeny of the catalytic domains. Science 241: 42-52.

Harris, S.D., J.L. Morrell, and J.E. Hamer. 1994. Identification and characterization of Aspergillus nidulans mutants defective in cytokinesis. Genetics 136: 517-532.

Hartmann, H.A., R. Kahmann, and M. Bolker. 1996. The pheromone response factor coordinates filamentous growth and pathogenicity in Ustilago maydis. EMBO J. 15: 1632-1641.

Herskowitz, I. 1995. MAP kinase pathways in yeast: For mating and more. Cell 80: 187-197.

Hoch, H.C. and R.C. Staples. 1991. Signaling for infection structure formation in fungi. In Fungal spore and disease initiation in plants and animals (ed. G.T. Cole and H.C. Hoch), pp. 25-46. Plenum, New York, NY.

Howard, R.J. 1994. Cell biology of pathogenesis. In Rice blast disease (ed. R.S. Zeigler, S.A. Leong, and P.S. Teng), pp. 3-22. $\mathrm{CAB}$ International, Wallingford, UK.
Howard, R.J., M.A. Ferrari, D.H. Roach, and N.P. Money. 1991. Penetration of hard substrates by a fungus employing enormous turgor pressures. Proc. Natl. Acad. Sci. 88: 1128111284.

Huxley, H.E., E.D. Green, and I. Dunham. 1990. Rapid assessment of $S$. cerevisiae mating type by PCR. Trends Genet. 6: 236.

Kwon, Y.H., H.C. Hoch, and R.C. Staples. 1991. Cytoskeletal organization in Uromyces urediospore germling apices during appressorium formation. Protoplasma 165: 37-50.

Krisak, L., R. Strich, R.S. Winters, J.P. Hall, M.J. Mallory, D. Krietzer, R.S. Tuan, and E. Winter. 1994. SMK1, a developmentally regulated MAP kinase, is required for spore wall assembly in Saccharomyces cerevisiae. Genes \& Dev. 8: 2151-2161.

Lau, G. and J.E. Hamer. 1996. Genetic identification of two regulatory loci controlling metabolism and pathogenicity gene expression in the rice blast fungus. Plant Cell 8: 771781.

Lee, K.S., K. Irie, Y. Gotoh, Y. Watanabe, H. Araki, E. Nishida, K. Matsumoto, and D.E. Levin. 1993. A yeast mitogen-activated protein kinase homolog (Mpklp) mediates signaling by protein kinase C. Mol. Cell. Biol. 13: 3067-3075.

Lee, Y.H. and R.A. Dean. 1993a. cAMP regulates infection structure formation in the plant pathogenic fungus Magnaporthe grisea. Plant Cell 5: 693-700.

-1993b. Stage-specific gene expression during appressorium formation of Magnaporthe grisea. Exp. Mycol. 17: 215-222.

-1994. Hydrophobicity of contact surface induces appressorium formation of Magnaporthe grisea. FEMS Microbiol. Letts. 115: 71-76.

Leung, H. and P.H. Williams. 1985. Genetic analyses of electrophoretic enzyme variants, mating type, and hermaphroditism in Pyricularia oryzae. Can. J. Genet. Cytol. 27: 697-704.

Levy, M., J. Romao, M.A. Marchetti, and J.E. Hamer. 1991. DNA fingerprinting resolves pathotype diversity in a plant pathogenic fungus. Plant Cell 3: 95-102.

Ma, D., J.G. Cook, and J. Thorner. 1995. Phosphorylation and localization of Kss1, a MAP kinase of the Saccharomyces cerevisiae pheromone response pathway. Mol. Biol. Cell 6: 889-909.

Mitchell, T.K. and R.A. Dean. 1995. The cAMP-dependent protein kinase catalytic subunit is required for appressorium formation and pathogenesis by the rice blast pathogen $\mathrm{Mag}$ naporthe grisea. Plant Cell 7: 1869-1878.

Nishida, E. and Y. Gotoh. 1993. The MAP kinase cascade is essential for diverse signal transduction pathways. Trends Biochem. Sci. 18: 128-131.

Notteghem, J.L. and D. Silue. 1991. Distribution of the mating type alleles in Magnaporthe grisea populations pathogenic on rice. Phytopathology 82: 421-424.

Ou, S.H. 1985. Rice diseases. Commonwealth Mycological Institute, Surrey, UK.

Rhodes, N., L. Connell, and B. Errede. 1990. STE1l is a protein kinase required for cell-type-specific transcription and signal transduction in yeast. Genes \& Dev. 4: 1862-1874.

Romao, J. and J.E. Hamer. 1992. Genetic organization of a repeated DNA sequence family in the rice blast fungus. Proc. Natl. Acad. Sci. 89: 5316-5320.

Sambrook, J., E.F. Fritsch, and T. Maniatis. 1989. Molecular cloning: A laboratory manual, 2nd ed. Cold Spring Harbor Laboratory Press, Cold Spring Harbor, NY.

Sprague, G.F. 1991. Assay of yeast mating reaction. In Methods in enzymology (ed. C. Guthrie and G.R. Fink), Vol. 194, pp. 77-93. Academic Press, San Diego, CA. 
Smith, D.B. and K.S. Johnson. 1988. Single step purification of polypeptides expressed in Escherichia coli as fusions with glutathione S-transferase. Gene 67: 31-40.

Staben, C. 1995. Sexual reproduction in higher fungi. In The growing fungus (ed. N.A.R. Gow and G.M. Gadd), pp. 383402. Chapman and Hall, London, UK.

St. Leger, R.J., T.M. Butt, R.C. Staples, and D.W. Roberts. 1990. Second messenger involvement in differentiation of the entomopathogenic fungus Metarhizium anisopliae. I. Gen. Microbiol. 136: 1779-1790.

Sweigard, J.A., F.G. Chumley, and B. Valent. 1992. Cloning and analysis of Cut1, a cutinase gene from Magnaporthe grisea. Mol. Gen. Genet. 232: 174-182.

Talbot, N.J., D.J. Ebbole, and J.E. Hamer. 1993. Identification and characterization of MPG1, a gene involved in pathogenicity from the rice blast fungus Magnaporthe grisea. Plant Cell 5: 1575-1590.

Teng, P.S. 1994. The epidemiological basis for blast management. In Rice blast disease (ed. R.S. Zeigler, S.A. Leong, and P.S. Teng), pp. 409-434. CAB International, Wallingford, UK.

Thevelein, J.M. 1994. Signal transduction in yeast. Yeast 10: $1753-1790$.

Timberlake, W.E. 1980. Developmental gene regulation in Aspergillus nidulans. Dev. Biol. 78: 497-510.

Valent, B. 1990. Rice blast as a model system for plant pathology. Phytopathology 80: 33-36.

Valent, B., L. Farral, and F.G. Chumley. 1991. Magnaporthe grisea genes for pathogenicity and virulence identified through a series of backcrosses. Genetics 127: 87-101.

van der Plaat, J.B. 1974. Cyclic 3', 5'-adenosine monophosphate stimulates trehalose degradation in baker's yeast. Biochem. Biophys. Res. Commun. 56: 580-587.

$\mathrm{Xu}, \mathrm{J} . \mathrm{R}$. and J.E. Hamer. 1995. Assessment of Magnaporthe grisea mating type by spore-PCR. Fung. Genet. Newsl. 40: 80 .

Xu, J.R. and J.F. Leslie. 1996. A RFLP map of Gibberella fujikuroi mating population A. Genetics 143: 175-189. 


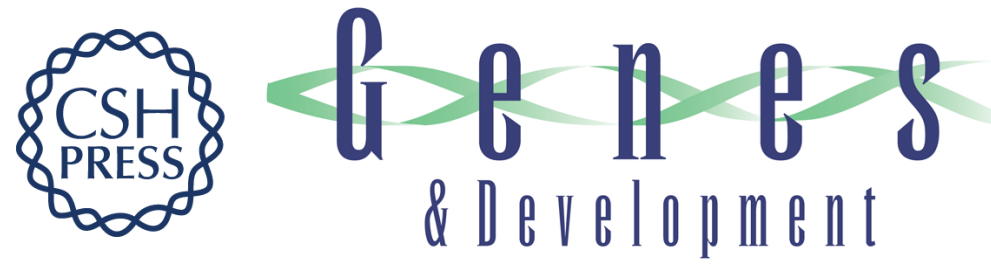

\section{MAP kinase and cAMP signaling regulate infection structure formation and pathogenic growth in the rice blast fungus Magnaporthe grisea.}

J R Xu and J E Hamer

Genes Dev. 1996, 10:

Access the most recent version at doi:10.1101/gad.10.21.2696

References This article cites 46 articles, 19 of which can be accessed free at:

http://genesdev.cshlp.org/content/10/21/2696.full.html\#ref-list-1

License

Email Alerting Service

Receive free email alerts when new articles cite this article - sign up in the box at the top right corner of the article or click here.

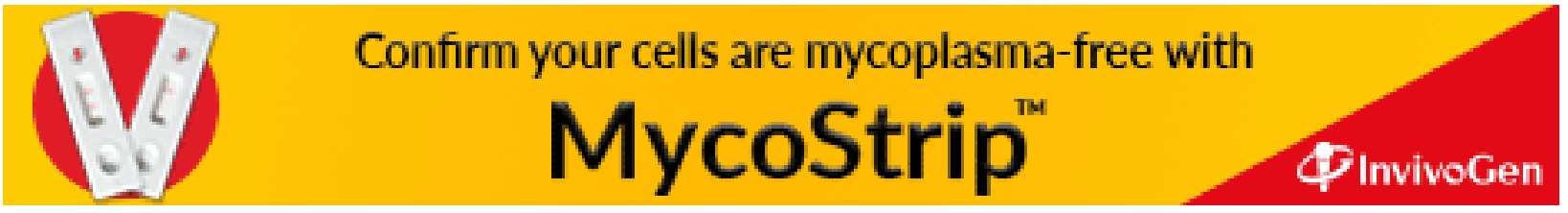

